\title{
QUASISTATIC DYNAMICS WITH INTERMITTENCY
}

\author{
JUHO LEPPÄNEN AND MIKKO STENLUND
}

\begin{abstract}
We study an intermittent quasistatic dynamical system composed of nonuniformly hyperbolic Pomeau-Manneville maps with time-dependent parameters. We prove an ergodic theorem which shows almost sure convergence of time averages in a certain parameter range, and identify the unique physical family of measures. The theorem also shows convergence in probability in a larger parameter range. In the process, we establish other results that will be useful for further analysis of the statistical properties of the model.
\end{abstract}

Acknowledgements. This work was supported by the Jane and Aatos Erkko Foundation, and by Emil Aaltosen Säätiö.

\section{INTRODUCTION}

In recent years time-dependent dynamical systems have gained an increasing amount of attention in the mathematics - especially mathematical physics - literature; see, for instance, $[1-3,5-8,11,12,15,16]$. On the other hand, nonuniformly hyperbolic dynamical systems with neutral fixed points have been studied extensively at least since the Pomeau-Manneville map was proposed as a model of the intermittent behavior of turbulent fluids $[4,13]$. In this paper we introduce a class of intermittent quasistatic dynamical systems, which combines the two, and initiate the study of their statistical properties. We begin the section by introducing the constituent intermittent dynamics and then proceed to defining their quasistatic conglomerate system.

Notations and conventions. The sigma-algebra on a topological space will always be that of the Borel sets. We denote the Lebesgue measure on $[0,1]$ by $m$ and write $m(f)=\int f d m=\int f d x$ for the Lebesgue integral of a function $f:[0,1] \rightarrow \mathbb{R}$, as well as $\int_{a}^{b} f d x=m\left(f 1_{[a, b]}\right)$ for $0 \leq a \leq b \leq 1$. The $L^{p}$ spaces are defined in terms of the Lebesgue measure. In particular, $\|f\|_{1}=\int|f| d x$. The space of continuous functions from $[0,1]$ to $\mathbb{R}$, denoted by $C([0,1])$, is equipped with the uniform norm and the resulting Borel sigma-algebra.

We use the usual floor and ceiling functions for rounding real numbers $x$ to the nearest integer:

$$
\lfloor x\rfloor=\max \{n \in \mathbb{N}: n \leq x\} \quad \text { and } \quad\lceil x\rceil=\min \{n \in \mathbb{N}: n \geq x\}
$$

System constants, whose values are determined solely by the parameters defining the model, are distinguished by a subindex (e.g., $C_{0}$ ) from generic constants (e.g., $C$ ), whose values may change from one expression to the next.

Key words and phrases. Quasistatic dynamical system, intermittency, Pomeau-Manneville map, ergodic theorem, physical family of measures.

2010 Mathematics Subject Classification. 37C60; 37D25, 37A10, 37A30. 
1.1. A class of intermittent maps. As in [9], let us define, for each $\alpha \in(0,1)$, the map $T_{\alpha}:[0,1] \rightarrow[0,1]:$

$$
T_{\alpha}(x)= \begin{cases}x\left(1+2^{\alpha} x^{\alpha}\right) & x \in\left[0, \frac{1}{2}\right) \\ 2 x-1 & x \in\left[\frac{1}{2}, 1\right] .\end{cases}
$$

It follows from [9] that $T_{\alpha}$ has an invariant SRB (Sinai-Ruelle-Bowen) measure $\hat{\mu}_{\alpha}$. This probability measure is equivalent to the Lebesgue measure $m$. We denote the density by $\hat{h}_{\alpha}$.

The word "intermittent" derives from the dynamical characteristics of such maps: The dynamics is strongly chaotic due to expansion except near the neutral (or indifferent) fixed point at the origin, where the derivative equals 1 . Once the orbit lands near the origin, it stays in its small neighborhood for a very long time before expansion has a noticeable effect again. The parameter $\alpha$ dictates how neutral the fixed point is; the larger $\alpha$ the longer it takes for the orbit to return to the expanding region of the interval. Consequently, correlation functions decay at a slower and slower rate (roughly as $n^{-\left(\frac{1}{\alpha}-1\right)}$, where $n$ is the time) and the invariant density becomes more and more concentrated around the origin as $\alpha$ increases (with an estimate $\hat{h}_{\alpha}(x) \lesssim x^{-\alpha}$ ). In the parameter range $0<\alpha<1$ studied here, the invariant density is nevertheless integrable. Pomeau-Manneville-type maps were originally proposed as models of the intermittent behavior observed in studies of turbulent fluids; see $[4,13]$. Since then, such maps have been investigated to the extent that we do not even attempt to cover the liteature here, besides the references cited below.

1.2. Quasistatic dynamical systems. The notion of an abstract quasistatic dynamical system, which we now recall, was introduced in [3]. Discussions on its physical interpretation and significance can be found in $[3,14]$.

Definition 1.1. Let $(X, \mathscr{F})$ be a measurable space, $\mathcal{M}$ a topological space whose elements are measurable self-maps $T: X \rightarrow X$, and $\mathbf{T}$ a triangular array of the form

$$
\mathbf{T}=\left\{T_{n, k} \in \mathcal{M}: 0 \leq k \leq n, n \geq 1\right\} .
$$

If there exists a piecewise continuous curve $\Gamma:[0,1] \rightarrow \mathcal{M}$ such that

$$
\lim _{n \rightarrow \infty} T_{n,\lfloor n t\rfloor}=\Gamma_{t}
$$

we say that $(\mathbf{T}, \Gamma)$ is a quasistatic dynamical system (QDS) with state space $X$ and system space $\mathcal{M}$.

Before describing the dynamics, let us define the intermittent QDS promised.

Definition 1.2 (An intermittent QDS). Let $X=[0,1]$ and $\mathcal{M}=\left\{T_{\alpha}: 0 \leq \alpha \leq 1\right\}$ (equipped, say, with the uniform topology). Next, let

$$
\left\{\alpha_{n, k} \in[0,1]: 0 \leq k \leq n, n \geq 1\right\}
$$

be a triangular array of parameters and

$$
\gamma:[0,1] \rightarrow[0,1]
$$

a piecewise continuous curve satisfying

$$
\lim _{n \rightarrow \infty} \alpha_{n,\lfloor n t\rfloor}=\gamma_{t}
$$

Finally, define $\Gamma_{t}=T_{\gamma_{t}}$ and

$$
T_{n, k}=T_{\alpha_{n, k}}
$$


It will often be convenient to use the parameter representation instead of the systemspace representation, i.e., to refer to $\alpha_{n, k}$ and $\gamma$ instead of $T_{n, k}$ and $\Gamma$.

The time evolution of an initial state $x \in[0,1]$ is now given by the triangular array $\mathbf{T}$, separately on each level of the array. That is, given $n \geq 1$, the point

$$
x_{n, k}=T_{n, k} \circ \cdots \circ T_{n, 1}(x) \in[0,1]
$$

is the state after $k \in\{0, \ldots, n\}$ steps on the $n$th level of $\mathbf{T}$. (We define $x_{n, 0}=x$.)

Introducing the continuous parameter $t \in[0,1]$ and setting $k=\lfloor n t\rfloor$, the piecewise constant curve $t \mapsto \alpha_{n,\lfloor n t\rfloor}$ approximates $\gamma$ with ever-increasing accuracy, as $n \rightarrow \infty$. In the physics terminology, it is helpful to think of $t$ as macroscopic time and, with $n$ fixed, of $k=\lfloor n t\rfloor$ as the corresponding microscopic time.

Given a measurable function $f:[0,1] \rightarrow \mathbb{R}$, we denote

$$
f_{n, k}=f \circ T_{n, k} \circ \cdots \circ T_{n, 1}, \quad 0 \leq k \leq n .
$$

(We define $f_{n, 0}=f$.) We define the functions $S_{n}:[0,1] \times[0,1] \rightarrow \mathbb{R}$ by

$$
S_{n}(x, t)=\int_{0}^{n t} f_{n,\lfloor s\rfloor}(x) d s, \quad n \geq 1 .
$$

Note that, given $x$, the function $S_{n}(x, \cdot)$ is a piecewise linear interpolation of the Birkhofftype sums $\sum_{k=0}^{\lfloor n t\rfloor-1} f_{n, k}(x), t \in[0,1]$, and, as such, an element of $C([0,1])$. Next, we define

$$
\zeta_{n}(x, t)=n^{-1} S_{n}(x, t)=\int_{0}^{t} f_{n,\lfloor n s\rfloor}(x) d s .
$$

Given an initial distribution $\mu$ for $x$, we can view $\zeta_{n}$ as a random element of $C([0,1])$.

In this paper we are interested in identifying conditions under which

$$
\lim _{n \rightarrow \infty} \zeta_{n}=\zeta
$$

almost surely with respect to an initial measure, where $\zeta \in C([0,1])$ has the expression

$$
\zeta(t)=\int_{0}^{t} \hat{\mu}_{\gamma_{s}}(f) d s .
$$

For a heuristic argument why such a result might be true, see [14]. In the fully degenerate case, in which $T_{n, k}=T_{\alpha}$ for some $\alpha$ and all $k$ and $n$, Birkhoff's ergodic theorem guarantees that, given an $L^{1}$ function $f, \lim _{n \rightarrow \infty} \zeta_{n}(x, t)=t \int f d \hat{\mu}_{\alpha}$ for almost every $x$ with respect to the SRB measure $\hat{\mu}_{\alpha}$ (equivalently $m$ ), for all $t \in[0,1]$. Due to the lack of an invariant measure, our approach in the proper QDS setup is not based on Birkhoff's ergodic theorem.

The notion of a physical family of measures, proposed in [14], is closely related to the problem above. Specializing to the setting of the current paper, let us recall the definition.

Definition 1.3. Let $\mathscr{P}=\left(\mu_{t}\right)_{t \in[0,1]}$ be a one-parameter family of measures on $[0,1]$, and suppose that the map $t \mapsto \mu_{s}(f)$ is measurable for all bounded continuous functions $f:[0,1] \rightarrow \mathbb{R}$. Suppose there exists a measurable set $A \subset[0,1]$ with $m(A)>0$ such that

$$
\lim _{n \rightarrow \infty} \int_{0}^{t} f_{n,\lfloor n s\rfloor}(x) d s=\int_{0}^{t} \mu_{s}(f) d s, \quad t \in[0,1]
$$

holds for all $x \in A$ and all bounded continuous functions $f:[0,1] \rightarrow \mathbb{R}$. Then we say that $\mathscr{P}$ is a physical family of measures for the $Q D S$. The set $A$ is called a basin of $\mathscr{P}$. 
Thus, a physical family of measures captures, in the limit $n \rightarrow \infty$, the mean behavior of the observations $f_{n, k}(x), k \geq 0$, for a notable fraction of initial points $x$ with respect to the Lebesgue measure $m$, which we point out is not invariant in any sense.

1.3. Main theorem. The following theorem is the main result of our paper, which we choose to present here because it can now be understood without additional preparation. In the course of its proof, we establish other results, interesting in their own right, that will be beneficial to further research on the statistical properties of the intermittent QDS introduced above (or similar models).

Theorem 1.4. Suppose that the curve $\gamma:[0,1] \rightarrow[0,1]$ is piecewise Hölder continuous ${ }^{1}$ with exponent $\theta \in(0,1]$, that

$$
\overline{\gamma([0,1])} \subset\left[0, \beta_{*}\right]
$$

for some $\beta_{*} \in(0,1)$, and that

$$
\lim _{n \rightarrow \infty} n^{\theta} \sup _{t \in[0,1]}\left|\alpha_{n,\lfloor n t\rfloor}-\gamma_{t}\right|<\infty .
$$

(i) If $\beta_{*} \geq \frac{1}{2}$, then for each $f \in C([0,1])$,

$$
\lim _{n \rightarrow \infty} \sup _{t \in[0,1]}\left|\zeta_{n}(x, t)-\zeta(t)\right|=0
$$

in probability, with respect to the Lebesgue measure. That is,

$$
\lim _{n \rightarrow \infty} m\left(\sup _{t \in[0,1]}\left|\zeta_{n}(x, t)-\zeta(t)\right| \geq \varepsilon\right)=0
$$

for all $\varepsilon>0$.

(ii) If $\beta_{*}<\frac{1}{2}$, then (4) holds for almost every $x \in[0,1]$ with respect to the Lebesgue measure. The one-parameter family of measures $\mathscr{P}=\left(\hat{\mu}_{\gamma_{t}}\right)_{t \in[0,1]}$ is the unique physical family of measures, and it has a basin $A$ of full measure, i.e., $m(A)=1$.

The condition in (2) is needed to maintain uniform control in certain estimates, mainly on the polynomial correlation decay, while the convergence rate in (3) is the natural one suggested by the "equipartition" $\alpha_{n, k}=\gamma_{k n^{-1}}$. The assumption that the curve $\gamma$ is Hölder continuous has a physical motivation: it could, for instance, be a Brownian path, as a consequence of external forcing on the system, and allowing jumps to occur accommodates for rare, sudden, changes in the forcing. Regarding the uniqueness of the physical family of measures, we recall that two such families, $\left(\mu_{\gamma_{t}}\right)_{t \in[0,1]}$ and $\left(\mu_{\gamma_{t}}^{\prime}\right)_{t \in[0,1]}$, are considered the same if $\mu_{\gamma_{t}}=\mu_{\gamma_{t}}^{\prime}$ except for a zero-measure set of parameters $t$.

We reiterate that the rate of correlation decay for the intermittent QDS is polynomial. The essential distinction between cases (i) and (ii) is the summability of this rate in case (ii), which yields stronger results.

As a matter of fact, we prove a bit more regarding case (i). Namely, if $f \in C^{1}([0,1])$, there exists a subsequence $\left(n_{k}\right)_{k \geq 1}$ along which the convergence in (4) is almost sure and $\lim _{k \rightarrow \infty} n_{k+1} / n_{k}=1$. Thus, the sequence converges almost surely, save for gaps of sublinearly growing length. One would like to patch the gaps and promote the result to almost sure convergence of the entire sequence, say, with the aid of the Borel-Cantelli lemma. We have been unable to do so. Alternatively, one could directly try to appeal to a sufficiently general strong law of large numbers for triangular arrays of random variables,

\footnotetext{
${ }^{1}$ Precisely, there exists a finite partition of $[0,1]$ into disjoint intervals $I_{1}, \ldots, I_{m}$ such that $\gamma$ is Hölder continuous on each $I_{i}$ with exponent $\theta$.
} 
but we have failed to find a suitable one in the probability theory literature. Either way, the critical difficulties encountered in establishing almost sure convergence in case (i) have to do with the necessity of controlling

$$
\zeta_{n}(t)-\zeta_{m}(t)=\int_{0}^{t} f \circ T_{n,\lfloor n s\rfloor} \circ \cdots \circ T_{n, 1}-f \circ T_{m,\lfloor m s\rfloor} \circ \cdots \circ T_{m, 1} d s
$$

with $n \neq m$, which involves comparing different levels of the array $\mathbf{T}$, consisting of different maps.

In view of (2), let us also point out that the QDS changes its nature if the curve is allowed to meet the right-hand boundary of $[0,1]$. For instance, the invariant density of $T_{1}$ is non-integrable. Nevertheless, it is possible that the result remains true, even in the almost sure sense, if the curve makes some isolated visits to the boundary. As we have tried to convey above, investigating this admittedly interesting question would require a notable amount of technical innovation.

Similar results for certain uniformly hyperbolic QDSs, enjoying exponential correlation decay, were obtained in [14]. The most significant difference here is that the intermittent QDS is nonuniformly hyperbolic. Nonuniformly hyperbolic systems are inherently more difficult to analyze due to the intermittent character of the dynamics alluded to above. To make matters worse, the system at issue - as a QDS - is time dependent. The study of time-dependent Pomeau-Manneville maps was recently initiated in [1], where a statistical memory-loss result for compositions of such maps was established; that result will have a role in the proof of Theorem 1.4.

Finally, the polynomial rate of correlation decay discussed above will also have an impact on other kinds of limit theorems in the future, including the assortment of techniques suitable for their proofs. For instance, one expects a central limit theorem to hold (only) in case (ii).

How the paper is organized. In Section 2 we discuss technical facts needed for understanding the subsequent sections. In Section 3 we elaborate on the connection of two classes of functions appearing in the proofs; this section also facilitates bounding multiple correlation functions, which is carried out in Section 4. In Section 5 we study the parameter dependence of the transfer operator and of the SRB density. The proof of the main result, Theorem 1.4, is finally given in Section 6.

\section{Preliminaries}

We denote the transfer operator associated to $T_{\alpha}$ and the Lebesgue measure by $\mathcal{L}_{\alpha}$. That is,

$$
\mathcal{L}_{\alpha} f(x)=\sum_{y \in T_{\alpha}^{-1} x} \frac{f(y)}{T_{\alpha}^{\prime}(y)}=\frac{f\left(y_{\alpha}\right)}{T_{\alpha}^{\prime}\left(y_{\alpha}\right)}+\frac{f\left(\frac{x}{2}+\frac{1}{2}\right)}{2},
$$

where $y_{\alpha}=y_{\alpha}(x)$ is the preimage of $x$ under the left branch $\left.T_{\alpha}\right|_{\left[0, \frac{1}{2}\right)}$. The following result establishes an important invariance property for the transfer operator:

Lemma 2.1. Let $0<\beta<1$ and $a(\beta)=2^{\beta}(\beta+2)$. The transfer operator $\mathcal{L}_{\alpha}$ maps the convex cone

$$
\begin{aligned}
\mathcal{C}_{*}(\beta)=\left\{f \in C((0,1]) \cap L^{1}:\right. & f \geq 0, f \text { decreasing, } \\
& \left.x^{\beta+1} f \text { increasing, } f(x) \leq a(\beta) x^{-\beta} m(f)\right\}
\end{aligned}
$$

into itself, provided $0<\alpha \leq \beta$. 
For a proof of Lemma 2.1, see [9] for the original case of a single parameter $(\alpha=\beta)$ and [1] for the above adaptation to a range of parameters $(0<\alpha \leq \beta)$.

It follows from [9] that the SRB density of $T_{\alpha}$ satisfies

$$
\hat{h}_{\alpha} \in \mathcal{C}_{*}(\alpha) \text {. }
$$

Note that the cone is increasing, i.e., $\mathcal{C}_{*}(\alpha) \subset \mathcal{C}_{*}(\beta)$ if $\alpha \leq \beta$. In particular, $\hat{h}_{\alpha} \in \mathcal{C}_{*}(\beta)$ whenever $\alpha \leq \beta$.

We will always consider $\beta_{*} \in(0,1)$ fixed, and write

$$
a=a\left(\beta_{*}\right) \quad \text { and } \quad \mathcal{C}_{*}=\mathcal{C}_{*}\left(\beta_{*}\right)
$$

for brevity. We will call a sequence $\left(T_{\alpha_{i}}\right)_{i \geq 1}$ of Pomeau-Manneville maps an admissible sequence if $\alpha_{i} \leq \beta_{*}$ for all $i$. To keep notations simple, we write

$$
T_{i}=T_{\alpha_{i}} \quad \text { and } \quad \mathcal{L}_{i}=\mathcal{L}_{\alpha_{i}}
$$

for such a sequence. We also denote

$$
\widetilde{T}_{n, m}=T_{n} \circ \cdots \circ T_{m} \quad \text { and } \quad \widetilde{\mathcal{L}}_{n, m}=\mathcal{L}_{n} \cdots \mathcal{L}_{m}, \quad m<n,
$$

together with

$$
\widetilde{T}_{n}=T_{n} \circ \cdots \circ T_{1} \quad \text { and } \quad \widetilde{\mathcal{L}}_{n}=\mathcal{L}_{n} \cdots \mathcal{L}_{1} .
$$

Nearly all the results below apply for general admissible sequences, without additional restrictions on $\beta_{*}$. Only in Section 6 do we need to assume $\beta_{*}<\frac{1}{2}$ in order to check Condition (A) of Lemma 6.4, which requires a summable rate of correlation decay.

Let us already recall the following key estimate from [1]; see also [9] for a similar result in the case of a single map instead of a sequence.

Lemma 2.2. There exists a constant $C_{0}=C_{0}\left(\beta_{*}\right)>0$ such that the following holds. Let $\left(T_{i}\right)_{i \geq 1}$ be admissible and $f, g \in \mathcal{C}_{*}$ with $\int f d x=\int g d x$. Then, for all $n \geq 0$,

$$
\left\|\widetilde{\mathcal{L}}_{n}(f-g)\right\|_{1} \leq C_{0}\left(\|f\|_{1}+\|g\|_{1}\right) \rho(n)
$$

where

$$
\rho(n)=n^{-\left(\frac{1}{\beta_{*}}-1\right)}(\log n)^{\frac{1}{\beta_{*}}}, \quad n \geq 2,
$$

and $\rho(0)=\rho(1)=1$.

\section{3. $C^{1}$ FunCtions And The CONE $\mathcal{C}_{*}$}

Because of the invariance property (Lemma 2.1), much of the initial technical work is carried out for functions belonging to the cone $\mathcal{C}_{*}$. However, in applications a more familiar class of functions is preferred. The following lemma - which yields a recipe for passing results from $\mathcal{C}_{*}$ to $C^{1}$ - is (essentially) from [9]. Since a detailed proof seems not to have been published, we provide it below as community service.

Lemma 3.1. There exists a constant $C_{1}=C_{1}\left(\beta_{*}\right)>0$ such that the following holds. Suppose $A, B \geq 0$. There exist numbers $\lambda<0, \nu>0$ and $\delta>0$ such that

$$
(f+\lambda x+\nu) h+\delta \in \mathcal{C}_{*}
$$

with

$$
\|(f+\lambda x+\nu) h+\delta\|_{1} \leq C_{1} A B
$$

for every $f \in C^{1}([0,1])$ with $\|f\|_{C^{1}} \leq A$ and every $h \in \mathcal{C}_{*}$ with $m(h) \leq B$. In particular, $(\lambda x+\nu) h+\delta \in \mathcal{C}_{*}$ with $\|(\lambda x+\nu) h+\delta\|_{1} \leq C_{1} A B$. 
Remark 3.2. The proof shows that there exist system constants $\lambda_{1}<0, \nu_{1}>0$ and $\delta_{1}>0$ such that

$$
\lambda=A \lambda_{1}, \quad \nu=A \nu_{1}, \quad \text { and } \quad \delta=A B \delta_{1} .
$$

While it is true that a similar result can be obtained with $\lambda=\|f\|_{C_{1}} \lambda_{1}, \nu=\|f\|_{C_{1}} \nu_{1}$ and $\delta=\|f\|_{C_{1}} m(h) \delta_{1}$ without imposing bounds on $\|f\|_{C_{1}}$ and $m(h)$, there is some virtue in having the constants depend on the upper bound only. For example, in the current formulation the function $(\lambda x+\nu) h+\delta$ is automatically in the cone.

Proof of Lemma 3.1. First note that fixing

$$
\lambda \leq-\left\|f^{\prime}\right\|_{\infty}
$$

implies that $f+\lambda x$ is decreasing. The core idea of the proof is now that, for large $\nu>0$, the function

$$
\left(\frac{f+\lambda x}{\nu}+1\right) h
$$

is very close to $h \in \mathcal{C}_{*}$, and thus belongs to the cone after a small modification. To implement this idea rigorously, we write

$$
(f+\lambda x+\nu) h+\delta=\nu\left(\left(\frac{f+\lambda x}{\nu}+1\right) h+\frac{\delta}{\nu}\right)=\nu\left(g h+\delta^{\prime}\right),
$$

where

$$
g=\frac{f+\lambda x}{\nu}+1 \quad \text { and } \quad \delta^{\prime}=\frac{\delta}{\nu} .
$$

It now suffices to show that if $g \in C^{1}([0,1])$ is an arbitrary function such that $\|g-1\|_{C^{1}}$ is sufficiently small and $g^{\prime} \leq 0$, there exists $\delta^{\prime}>0$ such that

$$
\psi=g h+\delta^{\prime} \in \mathcal{C}_{*} .
$$

Step 1. Suppose $g \geq 0$ and $g^{\prime} \leq 0$. Then $\psi \geq 0$ and $\psi$ is decreasing.

Step 2. We identify a condition which guarantees that $x^{\alpha+1} \psi$ is increasing. To that end, let $x<y$ and observe that

$$
\begin{aligned}
& y^{\alpha+1} \psi(y)-x^{\alpha+1} \psi(x) \\
& =g(y) y^{\alpha+1} h(y)-g(x) x^{\alpha+1} h(x)+\delta^{\prime}\left(y^{\alpha+1}-x^{\alpha+1}\right) \\
& =g(y)\left[y^{\alpha+1} h(y)-x^{\alpha+1} h(x)\right]-(g(x)-g(y)) x^{\alpha+1} h(x)+\delta^{\prime}\left(y^{\alpha+1}-x^{\alpha+1}\right) \\
& \geq-(g(x)-g(y)) x^{\alpha+1} h(x)+\delta^{\prime}\left(y^{\alpha+1}-x^{\alpha+1}\right),
\end{aligned}
$$

where we used that $x^{\alpha+1} h$ is increasing and $g \geq 0$. Since $g$ is a decreasing $C^{1}$ function and $0 \leq h(x) \leq a x^{-\alpha} m(h)$,

$$
-(g(x)-g(y)) x^{\alpha+1} h(x) \geq-\left\|g^{\prime}\right\|_{\infty}(y-x) a x m(h) .
$$

On the other hand,

$$
y^{\alpha+1}-x^{\alpha+1}=(\alpha+1) \int_{x}^{y} \xi^{\alpha} d x \geq(\alpha+1)(y-x) x^{\alpha} \geq(\alpha+1)(y-x) x .
$$

Thus, we arrive at

$$
y^{\alpha+1} \psi(y)-x^{\alpha+1} \psi(x) \geq\left[-\left\|g^{\prime}\right\|_{\infty} a m(h)+\delta^{\prime}(\alpha+1)\right](y-x) x,
$$

which is $\geq 0$, provided that

$$
\delta^{\prime} \geq \frac{a}{\alpha+1} m(h)\left\|g^{\prime}\right\|_{\infty}
$$


Step 3. We identify a condition which guarantees $\psi(x) \leq a x^{-\alpha} m(\psi)$, the latter being equivalent to $g h(x)+\delta^{\prime} \leq a x^{-\alpha}\left(m(g h)+\delta^{\prime}\right)$. Let us assume throughout that $\|g-1\| \leq \frac{1}{3}$. Note that

$$
1-\|g-1\|_{\infty} \leq g \leq 1+\|g-1\|_{\infty} .
$$

Thus, using $h(x) \leq a x^{-\alpha} m(h)$,

$$
g h(x) \leq\left(1+\|g-1\|_{\infty}\right) a x^{-\alpha} m(h),
$$

which yields

$$
g h(x)+\delta^{\prime} \leq \frac{1+\|g-1\|_{\infty}}{1-\|g-1\|_{\infty}} a x^{-\alpha} m(g h)+\delta^{\prime}=\left(1+\frac{2\|g-1\|_{\infty}}{1-\|g-1\|_{\infty}}\right) a x^{-\alpha} m(g h)+\delta^{\prime},
$$

We now make the assumption

$$
\delta^{\prime} \geq \frac{4 a}{a-1} m(h)\|g-1\|_{\infty} .
$$

It yields

$$
\begin{aligned}
& \frac{2\|g-1\|_{\infty}}{1-\|g-1\|_{\infty}} a x^{-\alpha} m(g h)+\delta^{\prime} \\
& \leq 2\|g-1\|_{\infty} \frac{1+\|g-1\|_{\infty}}{1-\|g-1\|_{\infty}} a x^{-\alpha} m(h)+x^{-\alpha} \delta^{\prime} \\
& \leq\left(4\|g-1\|_{\infty} a m(h)+\delta^{\prime}\right) x^{-\alpha} \leq a x^{-\alpha} \delta^{\prime},
\end{aligned}
$$

which gives the desired bound $g h(x)+\delta^{\prime} \leq a x^{-\alpha}\left(m(g h)+\delta^{\prime}\right)$.

Final step. Let us revert to the function $f$ and translate the above conditions to conditions on $\lambda, \nu$ and $\delta$ via (5). First of all,

$$
\lambda \leq-\left\|f^{\prime}\right\|_{\infty} \quad \text { and } \quad \nu \geq\|f\|_{\infty}-\lambda \quad \Longrightarrow \quad g \geq 0 \quad \text { and } \quad g^{\prime} \leq 0 .
$$

Secondly,

Finally,

$$
\nu \geq 3\left(\|f\|_{\infty}-\lambda\right) \quad \Longrightarrow \quad\|g-1\|_{\infty} \leq \frac{1}{3}
$$

and

$$
\delta \geq \frac{a}{\alpha+1} m(h)\left(\left\|f^{\prime}\right\|_{\infty}-\lambda\right) \quad \Longrightarrow \quad \delta^{\prime} \geq \frac{a}{\alpha+1} m(h)\left\|g^{\prime}\right\|_{\infty}
$$

$$
\delta \geq \frac{4 a}{a-1} m(h)\left(\|f\|_{\infty}-\lambda\right) \quad \Longrightarrow \quad \delta^{\prime} \geq \frac{4 a}{a-1} m(h)\|g-1\|_{\infty} .
$$

Choosing

$$
\lambda=-A, \quad \nu=6 A \quad \text { and } \quad \delta=2 A B \max \left(\frac{a}{\alpha+1}, \frac{4 a}{a-1}\right)
$$

is enough to satisfy all the conditions. Since

$$
\begin{aligned}
\|(f+\lambda x+\nu) h+\delta\|_{1} & \leq\left(\|f\|_{\infty}+|\lambda|+\nu\right) m(h)+\delta_{1} \\
& \leq(A+A+6 A) B+2 A B \max \left(\frac{a}{\alpha+1}, \frac{4 a}{a-1}\right),
\end{aligned}
$$

the proof is complete.

Let us immediately give an example of how Lemma 3.1 can be put to use. We will in fact need a more general result for our purposes, in order to prove certain correlation estimates later on. But proving the special case first already reveals the basic idea. 
Lemma 3.3. Let $\left(T_{i}\right)_{i \geq 1}$ be admissible, $f_{1}, f_{2} \in C^{1}([0,1]), h \in \mathcal{C}_{*}$ and $n \geq 0$. There exist $g_{1}, \ldots, g_{4} \in \mathcal{C}_{*}$ such that

$$
f_{2} \cdot \widetilde{\mathcal{L}}_{n}\left(f_{1} h\right)=g_{1}-g_{2}+g_{3}-g_{4}
$$

and

$$
\left\|g_{i}\right\|_{1} \leq C_{1}^{2}\left\|f_{1}\right\|_{C^{1}}\left\|f_{2}\right\|_{C^{1}} m(h)
$$

Proof. Let us write

$$
\widetilde{\mathcal{L}}_{n}\left(f_{1} h\right)=u_{1}-u_{2}
$$

with

$$
u_{1}=\widetilde{\mathcal{L}}_{n}\left[\left(f_{1}+\lambda_{1} x+\nu_{1}\right) h+\delta_{1}\right] \quad \text { and } \quad u_{2}=\widetilde{\mathcal{L}}_{n}\left[\left(\lambda_{1} x+\nu_{1}\right) h+\delta_{1}\right] .
$$

With $\lambda_{1}, \nu_{1}$ and $\delta_{1}$ as in Lemma 3.1, where we set $A=\left\|f_{1}\right\|_{C^{1}}$ and $B=m(h)$, the functions in the square brackets are in $\mathcal{C}_{*}$. Since $\mathcal{C}_{*}$ is preserved by $\widetilde{\mathcal{L}}_{n}$, also $u_{1}, u_{2} \in \mathcal{C}_{*}$. Moreover, there exists a system constant $C_{1}>0$ such that

$$
m\left(u_{i}\right) \leq C_{1}\left\|f_{1}\right\|_{C^{1} m}(h), \quad i=1,2 .
$$

We now apply Lemma 3.1 once more, with $A=\left\|f_{2}\right\|_{C^{1}}$ and $B=C_{1}\left\|f_{1}\right\|_{C^{1}} m(h)$ : There exist constants $\lambda_{2}, \nu_{2}$ and $\delta_{2}$ such that

$$
f_{2} u_{i}=\left[\left(f_{2}+\lambda_{2} x+\nu_{2}\right) u_{i}+\delta_{2}\right]-\left[\left(\lambda_{2} x+\nu_{2}\right) u_{i}+\delta_{2}\right], \quad i=1,2,
$$

where the functions in the square brackets are in $\mathcal{C}_{*}$ and the $L^{1}$ norm of each is bounded by $C_{1}^{2}\left\|f_{2}\right\|_{C^{1}}\left\|f_{1}\right\|_{C^{1}} m(h)$. The proof is complete.

The abovementioned necessary generalization of Lemma 3.3 is the following:

Theorem 3.4. Let $\left(T_{i}\right)_{i \geq 1}$ be admissible, $f_{1}, \ldots, f_{k} \in C^{1}([0,1]), h \in \mathcal{C}_{*}$ and $0 \leq n_{1} \leq$ $\cdots \leq n_{k}$. There exist functions $g_{i} \in \mathcal{C}_{*}$ and constants $\sigma_{i} \in \pm 1,1 \leq i \leq 2^{k}$, such that ${ }^{2}$

$$
f_{k} \widetilde{\mathcal{L}}_{n_{k-1}, n_{k-2}+1} \cdots f_{3} \widetilde{\mathcal{L}}_{n_{2}, n_{1}+1} f_{2} \widetilde{\mathcal{L}}_{n_{1}, 1} f_{1} h=\sum_{i=1}^{2^{k}} \sigma_{i} g_{i}
$$

with

$$
\left\|g_{i}\right\|_{1} \leq C_{1}^{k}\left\|f_{1}\right\|_{C^{1}} \cdots\left\|f_{k}\right\|_{C^{1}} m(h) .
$$

Proof. The proof proceeds by induction. By Lemma 3.3, the claim holds true for $k=2$. Suppose it holds true for some fixed $k \geq 2$ and note that

$$
f_{k+1} \widetilde{\mathcal{L}}_{n_{k}, n_{k-1}+1} \cdots f_{3} \widetilde{\mathcal{L}}_{n_{2}, n_{1}+1} f_{2} \widetilde{\mathcal{L}}_{n_{1}, 1} f_{1} h=\sum_{i=1}^{2^{k}} \sigma_{i} f_{k+1} \cdot \widetilde{\mathcal{L}}_{n_{k}, n_{k-1}+1}\left(g_{i}\right),
$$

where $\left\|\widetilde{\mathcal{L}}_{n_{k}, n_{k-1}+1} g_{i}\right\|_{1}=\left\|g_{i}\right\|_{1} \leq C_{1}^{k}\left\|f_{1}\right\|_{C^{1}} \cdots\left\|f_{k}\right\|_{C^{1}} m(h)$ holds. Mimicking the proof of Lemma 3.3, we apply Lemma 3.1 with $A=\left\|f_{k+1}\right\|_{C^{1}}$ and $B=C_{1}^{k}\left\|f_{1}\right\|_{C^{1}} \cdots\left\|f_{k}\right\|_{C^{1}} m(h)$ : There exist constants $\lambda, \nu$ and $\delta$ such that

$$
f_{k+1} \cdot \widetilde{\mathcal{L}}_{n_{k}, n_{k-1}+1} g_{i}=\left[\left(f_{k+1}+\lambda x+\nu\right) \widetilde{\mathcal{L}}_{n_{k}, n_{k-1}+1} g_{i}+\delta\right]-\left[(\lambda x+\nu) \widetilde{\mathcal{L}}_{n_{k}, n_{k-1}+1} g_{i}+\delta\right]
$$

for $1 \leq i \leq 2^{k}$, where the two functions in the square brackets are in $\mathcal{C}_{*}$ and the $L^{1}$ norm of each is bounded by $C_{1}\left\|f_{k+1}\right\|_{C^{1}} C_{1}^{k}\left\|f_{1}\right\|_{C^{1}} \cdots\left\|f_{k}\right\|_{C^{1}} m(h)$. This finishes the proof.

\footnotetext{
${ }^{2}$ In order to avoid cumbersome notation involving $k-1$ pairs of parentheses, the convention here is that each operator acts on the entire expression to its right.
} 


\section{Correlation DeCAY}

Given Theorem 3.4, we are now prepared to prove the following result on correlation decay. In addition to being necessary for our immediate needs, it will be applicable to proving limit theorems beyond this paper, such as a central limit theorem.

Theorem 4.1. Let $\left(T_{i}\right)_{i \geq 1}$ be admissible, $f_{0}, f_{1}, \ldots, f_{m} \in C^{1}([0,1])$ and $f_{m+1}, f_{m+2} \ldots, f_{k} \in$ $L^{\infty}$. Moreover, let $0 \leq n_{1} \leq \cdots \leq n_{k}$. Denote

$$
F_{m}=f_{m} \circ \widetilde{T}_{n_{m}} \cdots f_{1} \circ \widetilde{T}_{n_{1}} \cdot f_{0}
$$

and

$$
G_{m}=f_{k} \circ \widetilde{T}_{n_{k}} \cdots f_{m+1} \circ \widetilde{T}_{n_{m+1}} .
$$

Then

$$
\begin{aligned}
& \left|\int G_{m} F_{m} d \mu-\int G_{m} d \mu \int F_{m} d \mu\right| \\
& \leq 4 C_{0}\left(2 C_{1}\right)^{m+1}\left(\prod_{i=m+1}^{k}\left\|f_{i}\right\|_{\infty}\right)\left(\prod_{i=0}^{m}\left\|f_{i}\right\|_{C^{1}}\right) \rho\left(n_{m+1}-n_{m}\right)
\end{aligned}
$$

for any probability measure $d \mu=h d m$ with $h \in \mathcal{C}_{*}$.

Proof. First note that, writing

$$
\widetilde{G}_{m}=f_{k} \circ \widetilde{T}_{n_{k}, n_{m+1}+1} \cdot f_{k-1} \circ \widetilde{T}_{n_{k-1}, n_{m+1}+1} \cdots f_{m+1},
$$

we have $G_{m}=\widetilde{G}_{m} \circ \widetilde{T}_{n_{m+1}}$ and, for any constant $c$,

$$
\begin{aligned}
& \left|\int G_{m} F_{m} d \mu-\int G_{m} d \mu \int F_{m} d \mu\right|=\left|\int\left(G_{m}-\int G_{m} d \mu\right)\left(F_{m}-c\right) d \mu\right| \\
& =\left|\int\left(\widetilde{G}_{m}-\int G_{m} d \mu\right) \circ \widetilde{T}_{n_{m+1}}\left(F_{m}-c\right) d \mu\right| \\
& =\left|\int\left(\widetilde{G}_{m}-\int G_{m} d \mu\right) \widetilde{\mathcal{L}}_{n_{m+1}}\left(F_{m} h-c h\right) d x\right| \\
& \leq 2\left\|\widetilde{G}_{m}\right\|_{\infty}\left\|\widetilde{\mathcal{L}}_{n_{m+1}}\left(F_{m} h-c h\right)\right\|_{1} \\
& \leq 2\left(\prod_{i=m+1}^{k}\left\|f_{i}\right\|_{\infty}\right)\left\|\widetilde{\mathcal{L}}_{n_{m+1}, n_{m}+1}\left(\widetilde{\mathcal{L}}_{n_{m}}\left(F_{m} h\right)-c \tilde{h}\right)\right\|_{1}
\end{aligned}
$$

where we have introduced $\tilde{h}=\widetilde{\mathcal{L}}_{n_{m}} h \in \mathcal{C}_{*}$. Choosing

$$
c=\int \widetilde{\mathcal{L}}_{n_{m}}\left(F_{m} h\right) d x=\int F_{m} h d x
$$

guarantees

$$
\int\left(\widetilde{\mathcal{L}}_{n_{m}}\left(F_{m} h\right)-c \tilde{h}\right) d x=0 .
$$

Using the basic identity $\widetilde{\mathcal{L}}_{n}\left(f \circ \widetilde{T}_{n} g\right)=f \widetilde{\mathcal{L}}_{n} g$, note that

$$
\widetilde{\mathcal{L}}_{n_{i}}\left(F_{i} h\right)=\widetilde{\mathcal{L}}_{n_{i}}\left(f_{i} \circ \widetilde{T}_{n_{i}} F_{i-1} h\right)=f_{i} \widetilde{\mathcal{L}}_{n_{i}, n_{i-1}+1} \widetilde{\mathcal{L}}_{n_{i-1}}\left(F_{i-1} h\right) .
$$

By induction,

$$
\begin{aligned}
\widetilde{\mathcal{L}}_{n_{m}}\left(F_{m} h\right) & =f_{m} \widetilde{\mathcal{L}}_{n_{m}, n_{m-1}+1} \cdots f_{2} \widetilde{\mathcal{L}}_{n_{2}, n_{1}+1} \widetilde{\mathcal{L}}_{n_{1}}\left(F_{1} h\right) \\
& =f_{m} \widetilde{\mathcal{L}}_{n_{m}, n_{m-1}+1} \cdots f_{2} \widetilde{\mathcal{L}}_{n_{2}, n_{1}+1} f_{1} \widetilde{\mathcal{L}}_{n_{1}} f_{0} h .
\end{aligned}
$$


We can now apply Theorem 3.4, according to which

$$
\widetilde{\mathcal{L}}_{n_{m}}\left(F_{m} h\right)=\sum_{i=1}^{2^{m+1}} \sigma_{i} g_{i}
$$

for suitable functions $g_{i} \in \mathcal{C}_{*}$ and constants $\sigma_{i} \in \pm 1,1 \leq i \leq 2^{m+1}$, satisfying

$$
\left\|g_{i}\right\|_{1} \leq C_{1}^{m+1}\left\|f_{0}\right\|_{C^{1}} \cdots\left\|f_{m}\right\|_{C^{1}} .
$$

Writing $c_{i}=\int g_{i} d x$,

$$
\widetilde{\mathcal{L}}_{n_{m}}\left(F_{m} h\right)-c \tilde{h}=\sum_{i=1}^{2^{m+1}} \sigma_{i}\left(g_{i}-c_{i} \tilde{h}\right) .
$$

Each function in the parentheses on the right side satisfies the assumptions of Lemma 2.2. This yields

$$
\begin{aligned}
\left\|\widetilde{\mathcal{L}}_{n_{m+1}, n_{m}+1}\left(\widetilde{\mathcal{L}}_{n_{m}}\left(F_{m} h\right)-c \tilde{h}\right)\right\|_{1} & \leq \sum_{i=1}^{2^{m+1}}\left\|\widetilde{\mathcal{L}}_{n_{m+1}, n_{m}+1}\left(g_{i}-c_{i} \tilde{h}\right)\right\|_{1} \\
& \leq C_{0} \sum_{i=1}^{2^{m+1}}\left(\left\|g_{i}\right\|_{1}+\left|c_{i}\right|\right) \rho\left(d_{m}\right) \\
& \leq 2 C_{0} \sum_{i=1}^{2^{m+1}}\left\|g_{i}\right\|_{1} \rho\left(d_{m}\right) \\
& \leq 2 C_{0} 2^{m+1} C_{1}^{m+1}\left\|f_{0}\right\|_{C^{1}} \cdots\left\|f_{m}\right\|_{C^{1}} \rho\left(d_{m}\right) .
\end{aligned}
$$

Collecting the estimates proves the theorem.

\section{Perturbation of transfer operator And SRB Density}

Here we prove that the SRB density $\hat{h}_{\alpha}$ and the pushforward $\mathcal{L}_{\alpha} h$ of any initial density $h \in \mathcal{C}_{*}$ depend Hölder continuously on the parameter $\alpha$ in the $L^{1}$ norm. To the authors' knowledge these are new results.

Theorem 5.1. Let $0<\beta_{*}<1$. There exists a constant $C_{2}=C_{2}\left(\beta_{*}\right)>0$ such that

$$
\left\|\left(\mathcal{L}_{\alpha}-\mathcal{L}_{\beta}\right) h\right\|_{1} \leq C_{2}\|h\|_{1}(\beta-\alpha)^{\frac{1}{3}\left(1-\beta_{*}\right)}|\log (\beta-\alpha)| \quad \forall h \in \mathcal{C}_{*}
$$

and

$$
\left\|\hat{h}_{\alpha}-\hat{h}_{\beta}\right\|_{1} \leq C_{2}(\beta-\alpha)^{\frac{1}{3}\left(1-\beta_{*}\right)^{2}}|\log (\beta-\alpha)|^{\frac{1}{\beta_{*}}}
$$

hold whenever $0 \leq \alpha<\beta \leq \beta_{*}$.

We do not claim the result to be optimal regarding the exponents.

From Theorem 5.1 we immediately get

Corollary 5.2. Let $0<\beta_{*}<1$ and let $C_{2}>0$ be as in Theorem 5.1. For every bounded measurable function $f:[0,1] \rightarrow \mathbb{R}$,

$$
\left|\int f d \hat{\mu}_{\alpha}-\int f d \hat{\mu}_{\beta}\right| \leq C_{2}\|f\|_{\infty}\left|(\beta-\alpha)^{\frac{1}{3}\left(1-\beta_{*}\right)^{2}}\right| \log (\beta-\alpha)^{\frac{1}{\beta_{*}}}
$$

holds whenever $0 \leq \alpha<\beta \leq \beta_{*}$. 
Proof of Theorem 5.1. The proof is given in the same order as the claims in the theorem.

Part 1: perturbation of the transfer operator. Let us first assume $\|h\|_{1}=1$. Since the maps $T_{\alpha}$ and $T_{\beta}$ agree on $\left[\frac{1}{2}, 1\right]$,

$$
\left(\mathcal{L}_{\alpha}-\mathcal{L}_{\beta}\right) h(x)=\frac{h\left(y_{\alpha}\right)}{T_{\alpha}^{\prime}\left(y_{\alpha}\right)}-\frac{h\left(y_{\beta}\right)}{T_{\beta}^{\prime}\left(y_{\beta}\right)},
$$

where $y_{\alpha}$ is the preimage of $x$ under the left branch $\left.T_{\alpha}\right|_{\left[0, \frac{1}{2}\right)}$ and $y_{\beta}$ is defined similarly. We begin by fixing $\varepsilon \in(0,1)$ and noting that

$$
\int_{[0, \varepsilon]} \frac{h\left(y_{\alpha}\right)}{T_{\alpha}^{\prime}\left(y_{\alpha}\right)} d x=\int_{\left\{y: T_{\alpha}(y) \in[0, \varepsilon]\right\}} h(y) d y \leq \int_{[0, \varepsilon]} h(y) d y \leq C \varepsilon^{1-\beta_{*}},
$$

where the last inequality uses the fact that $h \in \mathcal{C}_{*}$, and the constant $C$ depends only on $\beta_{*}$. A similar bound holds for $y_{\beta}$ in place of $y_{\alpha}$ and $T_{\beta}$ in place of $T_{\alpha}$. Hence,

$$
\int_{[0, \varepsilon]}\left|\frac{h\left(y_{\alpha}\right)}{T_{\alpha}^{\prime}\left(y_{\alpha}\right)}-\frac{h\left(y_{\beta}\right)}{T_{\beta}^{\prime}\left(y_{\beta}\right)}\right| d x \leq 2 C \varepsilon^{1-\beta} .
$$

We are left with an $L^{1}$ estimate on $(\varepsilon, 1]$. To that end, we bound

$$
\begin{aligned}
\left|\frac{h\left(y_{\alpha}\right)}{T_{\alpha}^{\prime}\left(y_{\alpha}\right)}-\frac{h\left(y_{\beta}\right)}{T_{\beta}^{\prime}\left(y_{\beta}\right)}\right| & =\left|\frac{h\left(y_{\alpha}\right)-h\left(y_{\beta}\right)}{T_{\alpha}^{\prime}\left(y_{\alpha}\right)}-\frac{h\left(y_{\beta}\right)}{T_{\beta}^{\prime}\left(y_{\beta}\right)} \frac{1}{T_{\alpha}^{\prime}\left(y_{\alpha}\right)}\left(T_{\alpha}^{\prime}\left(y_{\alpha}\right)-T_{\beta}^{\prime}\left(y_{\beta}\right)\right)\right| \\
& \leq\left|h\left(y_{\alpha}\right)-h\left(y_{\beta}\right)\right|+\frac{h\left(y_{\beta}\right)}{T_{\beta}^{\prime}\left(y_{\beta}\right)}\left|T_{\alpha}^{\prime}\left(y_{\alpha}\right)-T_{\beta}^{\prime}\left(y_{\beta}\right)\right| .
\end{aligned}
$$

Observe that

$$
T_{\alpha}^{\prime}\left(y_{\alpha}\right)=(1+\alpha) x y_{\alpha}^{-1}-\alpha
$$

where

$$
1 \leq x y_{\alpha}^{-1} \leq 2
$$

because $x=T_{\alpha}\left(y_{\alpha}\right)=y_{\alpha}\left(1+2^{\alpha} y_{\alpha}^{\alpha}\right)$. Using this together with $\left\|\mathcal{L}_{\beta} h\right\|_{1}=1$, we estimate

$$
\begin{aligned}
& \int_{(\varepsilon, 1]} \frac{h\left(y_{\beta}\right)}{T_{\beta}^{\prime}\left(y_{\beta}\right)}\left|T_{\alpha}^{\prime}\left(y_{\alpha}\right)-T_{\beta}^{\prime}\left(y_{\beta}\right)\right| d x \\
& \leq\left\|\mathcal{L}_{\beta} h\right\|_{1}\left\|\left((1+\alpha) x y_{\alpha}^{-1}-\alpha-(1+\beta) x y_{\beta}^{-1}+\beta\right) 1_{(\varepsilon, 1]}(x)\right\|_{\infty} \\
& \leq(\beta-\alpha)+\left\|\left((1+\alpha) x y_{\alpha}^{-1}-(1+\beta) x y_{\beta}^{-1}\right) 1_{(\varepsilon, 1]}(x)\right\|_{\infty} \\
& =(\beta-\alpha)+\left\|\left((1+\alpha)\left(x y_{\alpha}^{-1}-x y_{\beta}^{-1}\right)-(\beta-\alpha) x y_{\beta}^{-1}\right) 1_{(\varepsilon, 1]}(x)\right\|_{\infty} \\
& \leq 3(\beta-\alpha)+2\left\|\left(x y_{\alpha}^{-1}-x y_{\beta}^{-1}\right) 1_{(\varepsilon, 1]}(x)\right\|_{\infty} \\
& \leq 3(\beta-\alpha)+2\left\|x y_{\alpha}^{-1} x y_{\beta}^{-1} x^{-1}\left(y_{\alpha}-y_{\beta}\right) 1_{(\varepsilon, 1]}(x)\right\|_{\infty} \\
& \leq 3(\beta-\alpha)+8\left\|x^{-1}\left(y_{\alpha}-y_{\beta}\right) 1_{(\varepsilon, 1]}(x)\right\|_{\infty} \\
& \leq 3(\beta-\alpha)+8 \varepsilon^{-1}\left\|\left(y_{\alpha}-y_{\beta}\right) 1_{(\varepsilon, 1]}(x)\right\|_{\infty} .
\end{aligned}
$$

Note that, because $T_{\beta}$ is expanding,

$$
\left|y_{\beta}-y_{\alpha}\right| \leq\left|T_{\beta}\left(y_{\beta}\right)-T_{\beta}\left(y_{\alpha}\right)\right|=\left|T_{\alpha}\left(y_{\alpha}\right)-T_{\beta}\left(y_{\alpha}\right)\right| \text {. }
$$

On the other hand, $x \geq \varepsilon$ implies $y_{\alpha}, y_{\beta} \geq \frac{\varepsilon}{2}$, so

$$
\begin{aligned}
\left|T_{\alpha}\left(y_{\alpha}\right)-T_{\beta}\left(y_{\alpha}\right)\right| & =\left|2^{\alpha} y_{\alpha}^{\alpha+1}-2^{\beta} y_{\alpha}^{\beta+1}\right|=\frac{1}{2}\left|\left(2 y_{\alpha}\right)^{1+\alpha}-\left(2 y_{\alpha}\right)^{1+\beta}\right| \\
& \leq\left|\log \left(2 y_{\alpha}\right)\right||\beta-\alpha| \leq \log \left(\varepsilon^{-1}\right)(\beta-\alpha)
\end{aligned}
$$


The function $u(x)=x^{1+\beta_{*}} h(x)$ is Lipschitz continuous with a Lipschitz constant $C$ depending only on $\beta_{*}{ }^{3}$ For $x \geq \varepsilon$ (and $y_{\alpha}, y_{\beta} \geq \frac{\varepsilon}{2}$ ) this implies

$$
\begin{aligned}
& \left|h\left(y_{\alpha}\right)-h\left(y_{\beta}\right)\right| \\
& =\left|y_{\alpha}^{-1-\beta_{*}} u\left(y_{\alpha}\right)-y_{\beta}^{-1-\beta_{*}} u\left(y_{\beta}\right)\right| \\
& \leq\left|\left(y_{\alpha}^{-1-\beta *}-y_{\beta}^{-1-\beta *}\right) u\left(y_{\alpha}\right)\right|+\left|y_{\beta}^{-1-\beta}\left(u\left(y_{\beta}\right)-u\left(y_{\alpha}\right)\right)\right| \\
& \leq\left(C \varepsilon^{-2-\beta}+C \varepsilon^{-1-\beta}\right)\left|y_{\beta}-y_{\alpha}\right| \\
& \leq C \varepsilon^{-2-\beta}\left|y_{\beta}-y_{\alpha}\right|,
\end{aligned}
$$

where $C>0$ still depends only on $\beta_{*}$. We now have (with new constants)

$$
\left\|\left(\mathcal{L}_{\alpha}-\mathcal{L}_{\beta}\right) h\right\|_{1} \leq C\left(\varepsilon^{1-\beta}+(\beta-\alpha) \varepsilon^{-2-\beta} \log \varepsilon^{-1}\right) \leq C\left(\varepsilon^{1-\beta}+(\beta-\alpha) \varepsilon^{-2-\beta}\right) \log \varepsilon^{-1},
$$

and setting $\varepsilon=(\beta-\alpha)^{\frac{1}{3}}$ yields

$$
\left\|\left(\mathcal{L}_{\alpha}-\mathcal{L}_{\beta}\right) h\right\|_{1} \leq C(\beta-\alpha)^{\frac{1}{3}(1-\beta)}|\log (\beta-\alpha)| \leq C(\beta-\alpha)^{\frac{1}{3}\left(1-\beta_{*}\right)}|\log (\beta-\alpha)| .
$$

The case $\|h\|_{1} \neq 1$ is recovered by scaling, which finishes the proof of the first part.

Part 2: perturbation of the SRB density. Since $\mathcal{L}_{\alpha} \hat{h}_{\alpha}=\hat{h}_{\alpha}$ for each $\alpha$, we have, for any $n \geq 0$,

$$
\hat{h}_{\alpha}-\hat{h}_{\beta}=\mathcal{L}_{\alpha}^{n}\left(\hat{h}_{\alpha}-1\right)-\mathcal{L}_{\alpha}^{n}\left(\hat{h}_{\beta}-1\right)+\left(\mathcal{L}_{\alpha}^{n}-\mathcal{L}_{\beta}^{n}\right) \hat{h}_{\beta} .
$$

Here $\hat{h}_{\alpha}, \hat{h}_{\beta}$ and 1 are in $\mathcal{C}_{*}$ with $\int \hat{h}_{\alpha} d x=\int \hat{h}_{\beta} d x=1$, so Lemma 2.2 yields

$$
\left\|\mathcal{L}_{\alpha}^{n}\left(\hat{h}_{\alpha}-1\right)-\mathcal{L}_{\alpha}^{n}\left(\hat{h}_{\beta}-1\right)\right\|_{1} \leq 4 C_{0} n^{-\left(\frac{1}{\beta_{*}}-1\right)}(\log n)^{\frac{1}{\beta_{*}}},
$$

where the constant depends only on $\beta_{*}$. Since

$$
\left(\mathcal{L}_{\alpha}^{n}-\mathcal{L}_{\beta}^{n}\right) \hat{h}_{\beta}=\sum_{k=1}^{n} \mathcal{L}_{\alpha}^{k-1}\left(\mathcal{L}_{\alpha}-\mathcal{L}_{\beta}\right) \mathcal{L}_{\beta}^{n-k} \hat{h}_{\beta}=\sum_{k=1}^{n} \mathcal{L}_{\alpha}^{k-1}\left(\mathcal{L}_{\alpha}-\mathcal{L}_{\beta}\right) \hat{h}_{\beta}
$$

and $\mathcal{L}_{\alpha}$ is an $L^{1}$ contraction, we also have

$$
\left\|\left(\mathcal{L}_{\alpha}^{n}-\mathcal{L}_{\beta}^{n}\right) \hat{h}_{\beta}\right\|_{1} \leq n\left\|\left(\mathcal{L}_{\alpha}-\mathcal{L}_{\beta}\right) \hat{h}_{\beta}\right\|_{1} .
$$

Applying the first part of the theorem and collecting all the estimates, we arrive at

$$
\begin{aligned}
\left\|\hat{h}_{\alpha}-\hat{h}_{\beta}\right\|_{1} & \leq C n\left(n^{-\frac{1}{\beta_{*}}}(\log n)^{\frac{1}{\beta_{*}}}+(\beta-\alpha)^{\frac{1}{3}\left(1-\beta_{*}\right)}|\log (\beta-\alpha)|\right) \\
& \leq C n\left(n^{-\frac{1}{\beta_{*}}}+(\beta-\alpha)^{\frac{1}{3}\left(1-\beta_{*}\right)}\right) \max \left((\log n)^{\frac{1}{\beta_{*}}},|\log (\beta-\alpha)|\right),
\end{aligned}
$$

where $C$ depends on $\beta_{*}$ only. Choosing $n=\left\lfloor(\beta-\alpha)^{-\frac{1}{3} \beta_{*}\left(1-\beta_{*}\right)}\right\rfloor \geq 1$ yields

$$
\begin{aligned}
\left\|\hat{h}_{\alpha}-\hat{h}_{\beta}\right\|_{1} & \leq C(\beta-\alpha)^{-\frac{1}{3} \beta_{*}\left(1-\beta_{*}\right)}\left(n^{-\frac{1}{\beta_{*}}}+(\beta-\alpha)^{\frac{1}{3}\left(1-\beta_{*}\right)}\right) \max \left((\log n)^{\frac{1}{\beta_{*}}},|\log (\beta-\alpha)|\right) \\
& \leq C(\beta-\alpha)^{\frac{1}{3}\left(1-\beta_{*}\right)^{2}} \max \left(|\log (\beta-\alpha)|^{\frac{1}{\beta_{*}}},|\log (\beta-\alpha)|\right) \\
& \leq C(\beta-\alpha)^{\frac{1}{3}\left(1-\beta_{*}\right)^{2}}|\log (\beta-\alpha)|^{\frac{1}{\beta_{*}}}
\end{aligned}
$$

with $C$ depending on $\beta_{*}$ only, where the last estimate uses $\beta-\alpha \leq \beta_{*}<1$. This finishes the proof of the second part.

The proof of Theorem 5.1 is now complete.

\footnotetext{
${ }^{3}$ See the footnote in the proof of Lemma 2.3 in [9].
} 


\section{Proof of MAIN THEOREM}

We are now ready to enter the proof of Theorem 1.4. We begin by recording certain facts concerning general values $\beta_{*} \in(0,1)$, which are essential for the proof, in Section 6.1. Parts (i) and (ii) of the theorem are proved in Sections 6.2 and 6.3, respectively.

6.1. Preliminaries $\left(0<\beta_{*}<1\right)$. Notice first that it will be enough to establish the theorem for all $f \in C^{1}([0,1])$ : Since $C^{1}([0,1])$ is a subalgebra of $C([0,1])$ which contains the constant functions and separates points, it is dense by the Stone-Weierstrass theorem. Hence, given $g \in C([0,1])$ and $\varepsilon>0$, there exists $f \in C^{1}([0,1])$ such that

$$
\sup _{t \in[0,1]}\left|\int_{0}^{t} g_{n,\lfloor n s\rfloor}(x) d s-\int_{0}^{t} \hat{\mu}_{s}(g) d s\right| \leq \sup _{t \in[0,1]}\left|\int_{0}^{t} f_{n,\lfloor n s\rfloor}(x) d s-\int_{0}^{t} \hat{\mu}_{s}(f) d s\right|+\varepsilon
$$

holds for all $x$ and all $n$.

Let $\left\{I_{1}, \ldots I_{m}\right\}$ be the regularity partition of $[0,1]$ associated to $\gamma$. We may assume $0=\tau_{1}<\tau_{2}<\cdots<\tau_{m}<\tau_{m+1}=1$, where $\tau_{\ell}$ and $\tau_{\ell+1}$ are the endpoints of the interval $I_{\ell}$. There exists a constant $C_{\gamma}>0$ such that

$$
\left|\gamma_{t}-\gamma_{s}\right| \leq C_{\gamma}|t-s|^{\theta}
$$

holds for all $\tau_{\ell}<s, t<\tau_{\ell+1}$ and all $\ell$, and

$$
\sup _{t \in[0,1]}\left|\alpha_{n,\lfloor n t\rfloor}-\gamma_{t}\right| \leq C_{\gamma} n^{-\theta}
$$

holds for all $n \geq 1$. Since $\left|\alpha_{n, k}-\alpha_{n, j}\right| \leq\left|\alpha_{n, n k n^{-1}}-\gamma_{k n^{-1}}\right|+\left|\alpha_{n, n j n^{-1}}-\gamma_{j n^{-1}}\right|+\left|\gamma_{k n^{-1}}-\gamma_{j n^{-1}}\right|$, the bounds above imply that

$$
\left|\alpha_{n, k}-\alpha_{n, j}\right| \leq C_{\gamma} n^{-\theta}\left(2+|j-k|^{\theta}\right)
$$

whenever $n \tau_{\ell}<j, k<n \tau_{\ell+1}$ holds for some $\ell$.

Note that there exist $0<\beta_{*}<1$ such that $\gamma([0,1]) \subset\left[0, \beta_{*}\right]$ and $\alpha_{n, k} \in\left[0, \beta_{*}\right]$ for $0 \leq k \leq n$ and sufficiently large values of $n$. Since we are only interested in the limit $n \rightarrow \infty$, we will assume - without loss of generality - that the latter condition holds starting from $n=1$.

We will shortly need the next lemma. For brevity, denote $\mathcal{L}_{n, k}=\mathcal{L}_{\alpha_{n, k}}$ and $\hat{h}_{n, k}=\hat{h}_{\alpha_{n, k}}$.

Lemma 6.1. Let $\mu$ be a probability measure with density $h \in \mathcal{C}_{*}$, and let $\mu_{n, k}$ be its pushforward with density $h_{n, k}=\mathcal{L}_{n, k} \cdots \mathcal{L}_{n, 1} h$. There exist constants $c_{0}, c_{1}>0$ and $p_{0}, p_{1} \in(0,1)$ such that

$$
\left\|h_{n, k}-\hat{h}_{n, k}\right\|_{1} \leq c_{0} n^{-p_{0}}
$$

whenever $n\left(\tau_{\ell}+c_{1} n^{p_{1}-1}\right)<k<n \tau_{\ell+1}$ holds for some $\ell$. Moreover, given a bounded function $f:[0,1] \rightarrow \mathbb{R}$

$$
\left|\mu\left(f_{n,\lfloor n t\rfloor}\right)-\hat{\mu}_{\gamma_{t}}(f)\right| \leq c_{0}\|f\|_{\infty} n^{-p_{0}}
$$

whenever $\tau_{\ell}+c_{1} n^{p_{1}-1}<t<\tau_{\ell+1}$ holds for some $\ell$.

For the purposes of this paper, the error rate in (11) is unnecessary. Moreover, (10) is only used in the proof of (11). However, both bounds will be useful for establishing finer statistical properties of the intermittent QDS later on. Information on the systemparameter dependence of the constants can be extracted from the proof below, which we leave to the interested reader. 
Proof of Lemma 6.1. Since $\mathcal{L}_{n, k} \hat{h}_{n, k}=\hat{h}_{n, k}$, we have

$$
h_{n, k}-\hat{h}_{n, k}=\mathcal{L}_{n, k} \cdots \mathcal{L}_{n, k-K+1}\left(h_{n, k-K}-\hat{h}_{n, k}\right)+\left(\mathcal{L}_{n, k} \cdots \mathcal{L}_{n, k-K+1}-\mathcal{L}_{n, k}^{K}\right) \hat{h}_{n, k}
$$

whenever $1 \leq K<k$. In order to bound $h_{n, k}-\hat{h}_{n, k}$ in $L^{1}$, note that Lemma 2.2 implies

$$
\left\|\mathcal{L}_{n, k} \cdots \mathcal{L}_{n, k-K+1}\left(h_{n, k-K}-\hat{h}_{n, k}\right)\right\|_{1} \leq C_{0}\left(\left\|h_{n, k-K}\right\|_{1}+\left\|\hat{h}_{n, k}\right\|_{1}\right) \rho(K) \leq 2 C_{0} \rho(K)
$$

Recalling that $\mathcal{L}_{\alpha}$ is an $L^{1}$ contraction and using (6) in Theorem 5.1,

$$
\begin{aligned}
& \left\|\left(\mathcal{L}_{n, k} \cdots \mathcal{L}_{n, k-K+1}-\mathcal{L}_{n, k}^{K}\right) \hat{h}_{n, k}\right\|_{1} \\
& =\left\|\sum_{j=k-K+1}^{k} \mathcal{L}_{n, k} \cdots \mathcal{L}_{n, j+1}\left(\mathcal{L}_{n, j}-\mathcal{L}_{n, k}\right) \mathcal{L}_{n, k}^{j-(k-K+1)} \hat{h}_{n, k}\right\|_{1} \\
& \leq \sum_{j=k-K+1}^{k}\left\|\left(\mathcal{L}_{n, j}-\mathcal{L}_{n, k}\right) \hat{h}_{n, k}\right\|_{1} \leq K \max _{k-K+1 \leq j \leq k}\left\|\left(\mathcal{L}_{n, j}-\mathcal{L}_{n, k}\right) \hat{h}_{n, k}\right\|_{1} \\
& \leq C_{2} K \max _{k-K+1 \leq j \leq k}\left|\alpha_{n, j}-\alpha_{n, k}\right|^{\frac{1}{3}\left(1-\beta_{*}\right)}|\log | \alpha_{n, j}-\alpha_{n, k} \| \\
& \leq C K \max _{k-K+1 \leq j \leq k}\left|\alpha_{n, j}-\alpha_{n, k}\right|^{\frac{1}{4}\left(1-\beta_{*}\right)}
\end{aligned}
$$

for a constant depending on $\beta_{*}$. Assuming

$$
n \tau_{\ell}+K<k<n \tau_{\ell+1}
$$

for some $\ell$, we can now use (9), which yields

$$
\begin{aligned}
& \left\|\left(\mathcal{L}_{n, k} \cdots \mathcal{L}_{n, k-K+1}-\mathcal{L}_{n, k}^{K}\right) \hat{h}_{n, k}\right\|_{1} \leq C K\left[C_{\gamma} n^{-\theta}\left(2+K^{\theta}\right)\right]^{\frac{1}{4}\left(1-\beta_{*}\right)} \\
& \leq C K\left[3 C_{\gamma} n^{-\theta} K^{\theta}\right]^{\frac{1}{4}\left(1-\beta_{*}\right)} \leq C n^{-\theta \frac{1}{4}\left(1-\beta_{*}\right)} K^{1+\theta \frac{1}{4}\left(1-\beta_{*}\right)}
\end{aligned}
$$

for constants $C$ depending only on $\beta_{*}$ and $\theta$ and $\gamma$. Writing $\kappa=\frac{1}{4}\left(1-\beta_{*}\right)$, we thus get

$$
\begin{aligned}
\left\|h_{n, k}-\hat{h}_{n, k}\right\|_{1} & \leq 2 C_{0} K^{-\left(\frac{1}{\beta_{*}}-1\right)}(\log K)^{\frac{1}{\beta_{*}}}+C n^{-\theta \kappa} K^{1+\theta \kappa} \\
& \leq C K\left(K^{\left.-\frac{1}{\beta_{*}}(\log K)^{\frac{1}{\beta_{*}}}+n^{-\theta \kappa} K^{\theta \kappa}\right)}\right.
\end{aligned}
$$

whenever (12) holds. Fixing $K=\left\lceil n^{\theta \kappa\left(\frac{1}{\beta_{*}}+\theta \kappa\right)^{-1}}\right\rceil$ (so that $K^{-\frac{1}{\beta_{*}}} \approx n^{-\theta \kappa} K^{\theta \kappa}$ ) yields

$$
\left\|h_{n, k}-\hat{h}_{n, k}\right\|_{1} \leq C n^{\theta \kappa\left(\frac{1}{\beta_{*}}+\theta \kappa\right)^{-1}\left(1-\frac{1}{\beta_{*}}\right)}(\log n)^{\frac{1}{\beta_{*}}} \leq c_{0} n^{-p_{0}},
$$

where $p_{0}$ depends on $\theta$ and $\beta_{*}$, and $c_{0}$ depends additionally on $\gamma$. This proves the first claim of the lemma, the values of $c_{1}$ and $p_{1}$ being determined by the choice of $K$.

In order to prove second claim, note first that $\mu\left(f_{n,\lfloor n t\rfloor}\right)=\mu_{n,\lfloor n t\rfloor}(f)$. Thus it suffices to bound $\left\|h_{n,\lfloor n t\rfloor}-\hat{h}_{\gamma_{t}}\right\|_{1}$. But, for $k=\lfloor n t\rfloor$ satisfying (12), we have

$$
\begin{aligned}
\left\|h_{n,\lfloor n t\rfloor}-\hat{h}_{\gamma_{t}}\right\|_{1} & \leq\left\|h_{n,\lfloor n t\rfloor}-\hat{h}_{n,\lfloor n t\rfloor}\right\|_{1}+\left\|\hat{h}_{n,\lfloor n t\rfloor}-\hat{h}_{\gamma_{t}}\right\|_{1} \\
& \leq c_{0} n^{-p_{0}}+C\left|\alpha_{n,\lfloor n t\rfloor}-\gamma_{t}\right|^{\frac{1}{4}\left(1-\beta_{*}\right)^{2}} \\
& \leq c_{0} n^{-p_{0}}+C n^{-\theta \frac{1}{4}\left(1-\beta_{*}\right)^{2}}
\end{aligned}
$$

with the aid of (7). The constant $C$ depends only on $\beta_{*}$ and $\theta$ and $\gamma$. Redefining $c_{0}$ and $p_{0}$ proves also the second claim. 
Our task is to bound

$$
\zeta_{n}(x, t)-\zeta(t)=\bar{\zeta}_{n}(x, t)+\int_{0}^{t} \mu\left(f_{n,\lfloor n s\rfloor}\right)-\hat{\mu}_{\gamma_{s}}(f) d s
$$

uniformly in $t$, where

$$
\bar{\zeta}_{n}(x, t)=\int_{0}^{t} \bar{f}_{n,\lfloor n s\rfloor}(x) d s \quad \text { and } \quad \bar{f}_{n,\lfloor n s\rfloor}(x)=f_{n,\lfloor n s\rfloor}(x)-\mu\left(f_{n,\lfloor n s\rfloor}\right) .
$$

Since $f$ is bounded, there are no integrability issues; in particular, Corollary 5.2 and the regularity of the curve $\gamma$ imply that $s \mapsto \hat{\mu}_{\gamma_{s}}(f)$ is piecewise continuous. We can proceed with an application of (11) in Lemma 6.1:

$$
\begin{aligned}
& \sup _{t \in[0,1]}\left|\int_{0}^{t} \mu\left(f_{n,\lfloor n s\rfloor}\right)-\hat{\mu}_{\gamma_{s}}(f) d s\right| \leq \int_{0}^{1}\left|\mu\left(f_{n,\lfloor n s\rfloor}\right)-\hat{\mu}_{\gamma_{s}}(f)\right| d s \\
& \quad=\sum_{\ell=1}^{m} \int_{\tau_{\ell}}^{\tau_{\ell}+c_{1} n^{p_{1}-1}}\left|\mu\left(f_{n,\lfloor n s\rfloor}\right)-\hat{\mu}_{\gamma_{s}}(f)\right| d s+\sum_{\ell=1}^{m} \int_{\tau_{\ell}+c_{1} n^{p_{1}-1}}^{\tau_{\ell+1}}\left|\mu\left(f_{n,\lfloor n s\rfloor}\right)-\hat{\mu}_{\gamma_{s}}(f)\right| d s \\
& \quad \leq 2\|f\|_{\infty} m c_{1} n^{p_{1}-1}+c_{0}\|f\|_{\infty} n^{-p_{0}},
\end{aligned}
$$

where the last bound tends to zero with increasing $n$.

Thus, it remains to bound $\bar{\zeta}_{n}$ in what follows. For convenience, we write $\bar{\zeta}_{n}(t)$ for the function $x \mapsto \bar{\zeta}_{n}(x, t)$. Roughly, the common strategy in the two cases of the theorem is to obtain moment bounds on $\zeta_{n}(t)$, for fixed values of $t$. The uniform Lipschitz continuity

$$
\left|\bar{\zeta}_{n}(x, t)-\bar{\zeta}_{n}(x, s)\right|=\left|\int_{s}^{t} \bar{f}_{n,\lfloor n r\rfloor} d r\right| \leq 2\|f\|_{\infty}|t-s|
$$

is then exploited for dealing with the uncountably many values $t$ can assume.

6.2. Case $\frac{1}{2} \leq \beta_{*}<1$ : proof of part (i). This case is based on the following second moment bound:

Lemma 6.2. Suppose $\beta_{*} \in\left[\frac{1}{2}, 1\right)$. Given $\delta \in\left(0, \frac{1}{\beta_{*}}-1\right)$, there exists a constant $C_{*}=$ $C_{*}\left(\beta_{*}, \delta\right)>0$ such that

$$
\mu\left(\left|\bar{\zeta}_{n}(t)\right|^{2}\right) \leq C_{*}\|f\|_{\infty}\|f\|_{C^{1} n^{-\delta}} t^{2-\delta}
$$

for all $n$ and $t .^{4}$

Proof. Fix $\delta \in\left(0, \frac{1}{\beta_{*}}-1\right)$ and define the auxiliary function

$$
\phi(u)= \begin{cases}u^{-\delta}, & u \geq 2 \\ 1, & 0 \leq u<2\end{cases}
$$

There exists a constant $C_{\phi}=C_{\phi}\left(\beta_{*}, \delta\right)$ such that $\rho(n) \leq C_{\phi} \phi(n)$ holds for all $n \geq 0$. Moreover, elementary computations show that

$$
\phi(\lfloor v\rfloor-\lfloor u\rfloor) \leq \phi\left(\frac{1}{2}(v-u)\right) \leq 8^{\delta} \phi(v-u), \quad u \leq v .
$$

\footnotetext{
${ }^{4}$ For completeness, we remark that if $\beta_{*} \in\left(0, \frac{1}{2}\right)$, the corresponding bound is $C\left(\beta_{*}\right)\|f\|_{\infty}\|f\|_{C^{1} n^{-1} t}$, which follows from the summability of $\rho$.
} 
Thus, by Theorem 4.1,

$$
\begin{aligned}
\mu\left(\left|\bar{\zeta}_{n}(t)\right|^{2}\right) & =\int_{0}^{t} \int_{0}^{t} \mu\left(\bar{f}_{n,\lfloor n s\rfloor} \bar{f}_{n,\lfloor n r\rfloor}\right) d r d s=2 \int_{0}^{t} \int_{0}^{s} \mu\left(\bar{f}_{n,\lfloor n s\rfloor} \bar{f}_{n,\lfloor n r\rfloor}\right) d r d s \\
& \leq 64 C_{0} C_{1} \int_{0}^{t} \int_{0}^{s}\left\|f-\mu\left(f_{n,\lfloor n s\rfloor}\right)\right\|_{\infty}\left\|f-\mu\left(f_{n,\lfloor n r\rfloor}\right)\right\|_{C^{1}} \rho(\lfloor n s\rfloor-\lfloor n r\rfloor) d r d s \\
& \leq 64 C_{0} C_{1} 8^{\delta} C_{\phi} 4\|f\|_{\infty}\|f\|_{C^{1}} \int_{0}^{t} \int_{0}^{s} \phi(n s-n r) d r d s \\
& =256 C_{0} C_{1} 8^{\delta} C_{\phi}\|f\|_{\infty}\|f\|_{C^{1}} \int_{0}^{t} \int_{0}^{s} \phi(n r) d r d s \\
& =256 C_{0} C_{1} 8^{\delta} C_{\phi}\|f\|_{\infty}\|f\|_{C^{1}} \cdot n^{-1} \int_{0}^{t} \int_{0}^{n s} \phi(r) d r d s \\
& \leq 256 C_{0} C_{1} 8^{\delta} C_{\phi}\|f\|_{\infty}\|f\|_{C^{1}} \cdot n^{-1} t \int_{0}^{n t} \phi(r) d r
\end{aligned}
$$

Assuming $n t \geq 2$,

$$
\int_{0}^{n t} \phi(r) d r=2+\frac{1}{1-\delta}\left((n t)^{1-\delta}-2^{1-\delta}\right) \leq 2+\frac{1}{1-\delta}(n t)^{1-\delta} \leq\left(2^{\delta}+\frac{1}{1-\delta}\right)(n t)^{1-\delta} .
$$

The same bound applies in the case $0 \leq n t<2$, as

$$
\int_{0}^{n t} \phi(r) d r=n t=(n t)^{\delta}(n t)^{1-\delta} \leq 2^{\delta}(n t)^{1-\delta} \leq\left(2^{\delta}+\frac{1}{1-\delta}\right)(n t)^{1-\delta} .
$$

Collecting the bounds proves the lemma.

Let $\left(K_{n}\right)_{n \geq 1}$ be an increasing sequence of positive integers, to be fixed later. Define the finite parameter sets

$$
\mathcal{T}_{n}=\left\{j K_{n}^{-1}: j=1, \ldots, K_{n}\right\}, \quad n \geq 1 .
$$

Given $t \in[0,1]$, let $t_{n}$ be the smallest element of $\mathcal{T}_{n}$ larger than $t$. Then

$$
\begin{aligned}
\sup _{t \in[0,1]}\left|\bar{\zeta}_{n}(t)\right| & \leq \sup _{t \in \mathcal{T}_{n}}\left|\bar{\zeta}_{n}(t)\right|+\sup _{t \in[0,1]}\left|\bar{\zeta}_{n}(t)-\bar{\zeta}_{n}\left(t_{n}\right)\right| \\
& \leq \sup _{t \in \mathcal{T}_{n}}\left|\bar{\zeta}_{n}(t)\right|+2\|f\|_{\infty} K_{n}^{-1} .
\end{aligned}
$$

Since $K_{n}$ is increasing, we thus have

$$
\limsup _{n \rightarrow \infty} \sup _{t \in[0,1]}\left|\bar{\zeta}_{n}(x, t)\right| \leq \limsup _{n \rightarrow \infty} \sup _{t \in \mathcal{T}_{n}}\left|\bar{\zeta}_{n}(x, t)\right|
$$

for every $x$. Next, following [10], we recall a Cauchy condensation criterion for the summability of a subsequence.

Lemma 6.3. Let the numbers $a_{n} \geq 0, n \geq 1$, satisfy

$$
\sum_{n=1}^{\infty} \frac{a_{n}}{n}<\infty \text {. }
$$

Then there exists an increasing sequence $\left(n_{k}\right)_{k \geq 1}$ such that

$$
\sum_{k=1}^{\infty} a_{n_{k}}<\infty \quad \text { and } \quad \lim _{k \rightarrow \infty} \frac{n_{k+1}}{n_{k}}=1 .
$$


We implement the lemma with $a_{n}=\mu\left(\left(\sup _{t \in \mathcal{T}_{n}}\left|\bar{\zeta}_{n}(t)\right|\right)^{2}\right)$. Observe that

$$
\mu\left(\left(\sup _{t \in \mathcal{T}_{n}}\left|\bar{\zeta}_{n}(t)\right|\right)^{2}\right)=\mu\left(\sup _{t \in \mathcal{T}_{n}}\left|\bar{\zeta}_{n}(t)\right|^{2}\right) \leq \sum_{t \in \mathcal{T}_{n}} \mu\left(\left|\bar{\zeta}_{n}(t)\right|^{2}\right)
$$

together with (13) yields

$$
\begin{aligned}
\sum_{n=1}^{\infty} \frac{1}{n} \mu\left(\left(\sup _{t \in \mathcal{T}_{n}}\left|\bar{\zeta}_{n}(t)\right|\right)^{2}\right) & \leq C_{*}\|f\|_{\infty}\|f\|_{C^{1}} \sum_{n=1}^{\infty} \frac{1}{n^{1+\delta}} \sum_{t \in \mathcal{T}_{n}} t^{2-\delta} \\
& \leq C_{*}\|f\|_{\infty}\|f\|_{C^{1}} \sum_{n=1}^{\infty} \frac{K_{n}}{n^{1+\delta}} .
\end{aligned}
$$

Choosing, say, $K_{n}=\lfloor\log n\rfloor$ guarantees that $\sum_{n=1}^{\infty} K_{n} / n^{1+\delta}<\infty$. By Lemma 6.3,

$$
\mu\left(\sum_{k=1}^{\infty}\left(\sup _{t \in \mathcal{T}_{n_{k}}}\left|\bar{\zeta}_{n_{k}}(t)\right|\right)^{2}\right)=\sum_{k=1}^{\infty} \mu\left(\left(\sup _{t \in \mathcal{T}_{n_{k}}}\left|\bar{\zeta}_{n_{k}}(t)\right|\right)^{2}\right)<\infty
$$

for some increasing sequence $\left(n_{k}\right)_{k \geq 1}$ satisfying $\lim _{k \rightarrow \infty} n_{k+1} / n_{k}=1$. This implies that

$$
\lim _{k \rightarrow \infty} \sup _{t \in \mathcal{T}_{n_{k}}}\left|\bar{\zeta}_{n_{k}}(x, t)\right|=0
$$

for almost every $x$ with respect to $\mu$. In view of (14),

$$
\lim _{k \rightarrow \infty} \sup _{t \in[0,1]}\left|\bar{\zeta}_{n_{k}}(t)\right|=0
$$

almost everywhere.

Finally, recall that a sequence of random variables converges in probability if and only if every subsequence has a further subsequence which converges almost surely. Repeating the above proof, mutatis mutandis, for an arbitrary subsequence $\sup _{t \in[0,1]}\left|\bar{\zeta}_{n_{k}}(t)\right|, k \geq 1$, yields a further subsequence $\sup _{t \in[0,1]}\left|\bar{\zeta}_{n_{k_{j}}}(t)\right|, j \geq 1$, which converges almost surely.

The proof of part (i) of Theorem 1.4 is now complete.

6.3. Case $\beta_{*}<\frac{1}{2}$ : proof of part (ii). In this case we cannot resort to the same method as in the previous one, because we want to prove almost sure convergence of the entire sequence, not just a subsequence. Following the approach of [14] for abstract QDSs, we take advantage of fourth moment bounds.

Given $f$ and $\mu$, let

$$
c_{n}^{\ell, j}\left(k_{1}, \ldots, k_{\ell}\right)=\mu\left(f_{n, k_{1}} \cdots f_{n, k_{\ell}}\right)-\mu\left(f_{n, k_{1}} \cdots f_{n, k_{j}}\right) \mu\left(f_{n, k_{j+1}} \cdots f_{n, k_{\ell}}\right)
$$

for all integers $2 \leq \ell \leq 4, j \in\{1, \ell-1\}$ and $k_{1}, \ldots, k_{\ell} \geq 0$. Note that if $c_{n}^{\ell, j}\left(k_{1}, \ldots, k_{\ell}\right)$ is small, then the products $f_{n, k_{1}} \cdots f_{n, k_{j}}$ and $f_{n, k_{j+1}} \cdots f_{n, k_{\ell}}$ are nearly uncorrelated with respect to the initial distribution $\mu$. We also introduce the function

$$
\Phi(s)= \begin{cases}s^{-1}(\log s)^{-2}, & s \geq 2 \\ 2^{-1}(\log 2)^{-2}, & 0 \leq s<2 .\end{cases}
$$

The key property of $\Phi$ is its integrability. 
Lemma 6.4. Let $f:[0,1] \rightarrow \mathbb{R}$ be a bounded measurable function and $\mu$ a probability measure on $[0,1]$. Suppose the following condition holds:

(A) There exists $C>0$ such that

$$
\left|c_{n}^{\ell, j}\left(k_{1}, \ldots, k_{\ell}\right)\right| \leq C \Phi\left(k_{j+1}-k_{j}\right)
$$

for all integers $2 \leq \ell \leq 4, j \in\{1, \ell-1\}$ and $0 \leq k_{1} \leq \cdots \leq k_{\ell}$.

Then

$$
\lim _{n \rightarrow \infty} \sup _{t \in[0,1]}\left|\bar{\zeta}_{n}(x, t)\right|=0
$$

for almost every $x$ with respect to $\mu$.

Proof. By Lemma 5.1 in [14], Condition (A) implies

$$
\sum_{n=1}^{\infty} \mu\left(\left|\bar{\zeta}_{n}(t)\right|^{4}\right)<\infty
$$

for all $t \in[0,1]$. In particular, given $t, \lim _{n \rightarrow \infty} \bar{\zeta}_{n}(x, t)=0$, for almost every $x$ with respect to $\mu$. Moreover, the functions $t \mapsto \bar{\zeta}_{n}(x, t)$ are uniformly Lipschitz continuous. By Lemma 4.1 in [14], $\lim _{n \rightarrow \infty} \sup _{t \in[0,1]}\left|\bar{\zeta}_{n}(x, t)\right|=0$.

It remains to check that Condition (A) holds with $f \in C^{1}([0,1])$ and $\mu=m$. To that end, we set $f_{0}=1$ and $f_{i}=f, i \geq 1$, in Theorem 4.1. This yields

$$
\begin{aligned}
\left|c_{n}^{\ell, j}\left(k_{1}, \ldots, k_{\ell}\right)\right| & \leq 4 C_{0}\left(2 C_{1}\right)^{j+1}\|f\|_{C^{1}}^{\ell} \rho\left(k_{j+1}-k_{j}\right) \\
& \leq 4 C_{0} \max \left(1,2 C_{1}\right)^{4} \max \left(1,\|f\|_{C^{1}}\right)^{4} \rho\left(k_{j+1}-k_{j}\right) .
\end{aligned}
$$

Since $\rho$ is dominated by $\Phi$ under the standing assumption $\beta_{*}<\frac{1}{2}$, we obtain $(\mathrm{A})$.

The result we have just proved also shows that the one-parameter family of measures $\mathscr{P}=\left(\hat{\mu}_{\gamma_{t}}\right)_{t \in[0,1]}$ is a physical family of measures with a basin of full measure. Its uniqueness follows from the latter property of the basin and Corollary 2.5 in [14].

The proof of part (ii), and thus of Theorem 1.4, is now complete.

\section{REFERENCES}

[1] Romain Aimino, Huyi Hu, Matthew Nicol, Andrei Török, and Sandro Vaienti. Polynomial loss of memory for maps of the interval with a neutral fixed point. Discrete Contin. Dyn. Syst., 35(3):793-806, 2015. Available from: http://dx.doi.org/10.3934/dcds.2015.35.793, doi:10.3934/dcds.2015.35.793.

[2] Jean-Pierre Conze and Albert Raugi. Limit theorems for sequential expanding dynamical systems on [0,1]. In Ergodic theory and related fields, volume 430 of Contemp. Math., pages 89-121. Amer. Math. Soc., Providence, RI, 2007. Available from: http://dx.doi.org/10.1090/conm/430/08253, doi : 10.1090/conm/430/08253.

[3] Neil Dobbs and Mikko Stenlund. Quasistatic dynamical systems. Submitted. Available from: http: //arxiv.org/abs/1504.01926.

[4] P. Gaspard and X.-J. Wang. Sporadicity: between periodic and chaotic dynamical behaviors. Proc. Nat. Acad. Sci. U.S.A., 85(13):4591-4595, 1988. Available from: http://dx.doi.org/10.1073/pnas.85.13.4591, doi:10.1073/pnas.85.13.4591.

[5] Chinmaya Gupta, William Ott, and Andrei Török. Memory loss for time-dependent piecewise expanding systems in higher dimension. Math. Res. Lett., 20(1):141-161, 2013. Available from: http://dx.doi.org/10.4310/MRL.2013.v20.n1.a12, doi:10.4310/MRL.2013.v20.n1.a12. 
[6] Christoph Kawan. Metric entropy of nonautonomous dynamical systems. Nonauton. Dyn. Syst., 1:26-52, 2014. Available from: http://dx.doi.org/10.2478/msds-2013-0003, doi: 10.2478/msds-2013-0003.

[7] Christoph Kawan. Expanding and expansive time-dependent dynamics. Nonlinearity, 28(3):669-695, 2015. Available from: http://dx.doi.org/10.1088/0951-7715/28/3/669, doi : $10.1088 / 0951-7715 / 28 / 3 / 669$.

[8] Andrzej Lasota and James A. Yorke. When the long-time behavior is independent of the initial density. SIAM J. Math. Anal., 27(1):221-240, 1996.

[9] Carlangelo Liverani, Benoît Saussol, and Sandro Vaienti. A probabilistic approach to intermittency. Ergodic Theory Dynam. Systems, 19(3):671-685, 1999. Available from: http://dx.doi.org/10.1017/S0143385799133856, doi:10.1017/S0143385799133856.

[10] Russell Lyons. Strong laws of large numbers for weakly correlated random variables. Michigan Math. J., 35(3):353-359, 1988. Available from: http://dx.doi.org/10.1307/mmj/1029003816, doi : $10.1307 / \mathrm{mmj} / 1029003816$.

[11] Péter Nándori, Domokos Szász, and Tamás Varjú. A Central Limit Theorem for Time-Dependent Dynamical Systems. Journal of Statistical Physics, 146(6):1213-1220, MAR 2012. Available from: http://dx.doi.org/10.1007/s10955-012-0451-8, doi:10.1007/s10955-012-0451-8.

[12] William Ott, Mikko Stenlund, and Lai-Sang Young. Memory loss for time-dependent dynamical systems. Mathematical Research Letters, 16(3):463-475, 2009. Available from: http: //www . aimsciences .org/journals/displayArticlesnew. jsp?paper ID=9775.

[13] Yves Pomeau and Paul Manneville. Intermittent transition to turbulence in dissipative dynamical systems. Comm. Math. Phys., 74(2):189-197, 1980. Available from: http://projecteuclid.org/euclid.cmp/1103907981.

[14] Mikko Stenlund. An almost sure ergodic theorem for quasistatic dynamical systems. Preprint. Available from: http://arxiv.org/abs/1507.02937.

[15] Mikko Stenlund. Non-stationary compositions of Anosov diffeomorphisms. Nonlinearity, 24:29913018, 2011. doi:doi:10.1088/0951-7715/24/10/016.

[16] Mikko Stenlund, Lai-Sang Young, and Hongkun Zhang. Dispersing billiards with moving scatterers. Commun. Math. Phys., 322(3):909-955, 2013. Available from: http://dx.doi.org/10.1007/s00220-013-1746-6.

(Juho Leppänen) Department of Mathematics and Statistics, P.O. Box 68, Fin-00014 UniVERSity OF HELSINKI, FinLAND.

E-mail address: juho.leppanen@helsinki.fi

(Mikko Stenlund) Department of Mathematics and Statistics, P.O. Box 68, Fin-00014 UniVERSiTy of Helsinki, Finland.

E-mail address: mikko.stenlund@helsinki.fi

URL: http://www.math.helsinki.fi/mathphys/mikko.html 